\title{
LES COMMUNAUTẼS D'OLIGOCHETES D'UN RUISSEAU DE PLAINE. LEUR UTILISATION COMME INDICATEURS DE LA POLLUTION ORGANIQUE
}

\author{
par Françoise Gross' ${ }^{1}$.
}

Les méthodes habituellement utilisées pour déterminer la qualité biologique d'un cours d'eau se sont avérées peu précises pour détecter la faible pollution organique de notre ruisseau.

Dans de telles conditions une méthode basée sur (1) la diversité spécifique des populations d'oligochètes, (2) le pourcentage d'oligochètes dans le benthos, (3) l'importance de l'association Tubifex tubifex (M.), Limnodrilus hoffmeisteri (Cl.) et L. udekamanius paraît mieux adaptée.

The oligochaete communities of a lowland stream and their value as indicators of organic pollution.

The usual methods used to determine the biological quality of running water are not very precise for the detection of mild pollution due to organic material from agriculture.

Under these conditions, the following criteria have been used, successfully : 1) the specific diversity of oligochaete populations, 2) the percentage of oligochaetes in the benthos, 3) the importance of the association Tubifex tubifex (M.), Limnodrilus hoffmeisteri (Cl.) and L. udekamanius.

\section{1. - INTRODUCTION}

La détermination de la qualité biologique des cours d'eau à l'aide des communautés d'invertébrés benthiques peut être effectuée selon différentes méthodes.

L'application des méthodes habituellement utilisées (saprobies de Kolkwitz et Marsson 1908-1909; indices biotiques de Tuffery et Verneaux 1967 ; indice de diversité de Shannon 1948) pour traduire graphiquement l'évolution de la pollution organique ne donnent pas toujours des résultats satisfaisants sur le cours d'eau étudié, soit parce qu'elles sont trop peu nuancées, soit parce qu'elles sont parfois même opposées à la réalité (Gross 1976).

1. Laboratoire d'anatomie comparée : Département Hydrobiologie, Université de Bordeaux I, avenue des Facultés, 33405 Talence Cedex. 
Une étude systématique des Oligochètes a donc été entreprise, ceux-ci ayant déjà été proposés par différents auteurs (Milbrink 1973 a et b) comme indicateurs de la qualité de l'eau.

Le but de cette étude est de chercher quelle est la précision des estimations de population qu'il est possible d'obtenir à partir du mode d'échantillonnage utilisé, de suivre les variations qualitatives et quantitatives des communautés d'Oligochètes en fonction de la pollution organique puis de proposer une méthode d'utilisation de ces communautés comme indicateurs de pollution.

\section{2. - MÉTHODES D'ÉCHANTILLONNAGE}

Huit séries de prélèvements de la faune benthique ont été effectuées de décembre 1973 à octobre 1974 sur 8 stations, à l'aide d'un

\begin{tabular}{|c|c|c|c|c|}
\hline Stations & Hiver & Printemps & Eté & Automne \\
\hline \multirow{3}{*}{1} & $457(215-975)$ & $776(539-1122)$ & $2399(1509-3821)$ & $3388(2017-5702)$ \\
\hline & Sur. : 5 u. e. & Car. : 10 u. e. & Car. : 10 u. e. & Car. : 10 u. e. \\
\hline & $69(28-171)$ & $933(569-1529)$ & $851(360-1906)$ & $794(513-1228)$ \\
\hline \multirow{2}{*}{2} & Sur. : 5 u. e. & Car. : 10 u. e. & Car. : 10 u. e. & Car. : 10 u. e. \\
\hline & $83(31-224)$ & $19(4-91)$ & $246(96-623)$ & $550(311-972)$ \\
\hline \multirow{2}{*}{31} & Sur. : 5 u. e. & Car. : 10 u. e. & Car.: 10 u. e. & Car. : 10 u. e. \\
\hline & $\mathbf{0}$ & 0 & $\mathbf{0}$ & $\mathbf{0}$ \\
\hline \multirow{2}{*}{$3^{\circ}$} & Sur. : 5 u. e. & Sur. : 5 u. e. & Sur. : 5 u. e. & Sur. : 5 u. e. \\
\hline & $7(0-102)$ & $20(8-57)$ & $10(2-28)$ & $5(0-22)$ \\
\hline \multirow{2}{*}{41} & Sur. : 5 u. e. & Sur. : 5 u. e. & Sur.: 5 u. e. & Sur. : 5 u. e. \\
\hline & $1(0-3)$ & 0 & $1(0-3)$ & $2(0-9)$ \\
\hline \multirow{2}{*}{$4^{e}$} & Sur. : 5 u. e. & Sur. : 5 u. e. & Sur. : 5 u. e. & Sur. : 5 u. e. \\
\hline & $324(45-2344)$ & $3890(1446-10471)$ & $6457(2882-14500)$ & $3631(653-20173)$ \\
\hline $5^{1}$ & Sur. : 3 u.e. & Car.: 3 u. e. & Car. : 3 u. e. & Car. : 3 u.e. \\
\hline \multirow[t]{2}{*}{$5^{e}$} & $195(87-437)$ & $3162(475-21057)$ & $537(5-19071)$ & $3802(837-17252)$ \\
\hline & Sur. : 3 u. e. & Car. : 3 u. e. & Car.: 3 u.e. & Car. : 3 u. e. \\
\hline
\end{tabular}

Tableau I, - Moyennes et limites de confiance au seuil de $95 \%$ des populations d'Oligochètes Tubificidae à chaque saison aux différentes stations. Pour chaque prélèvement, l'appareil utilisé (Carottier, Surber) et le nombre d'unités d'échantillonnage (u. e.) employées ont été précisés. 
filet de type Surber en faciès lotique et d'un carottier de $4 \mathrm{~cm}$ de diamètre s'enfonçant sur $5 \mathrm{~cm}$ en faciès lénitique (le nombre d'unités d'échantillonnage employées avec chaque appareil est précisé dans le tableau I).

En utilisant les méthodes préconisées par Elliott et Décamps (1973), nous avons calculé quelles étaient les moyennes des populations d'Oligochètes Tubificidae trouvées à chaque station et les limites de confiance de ces moyennes au seuil de $95 \%$.

Calcul des intervalles de confiance. Choix d'une méthode D'ÉCHANTILLONNAGE.

A chaque station, la distribution spatiale des Oligochètes étant du type contagieux (variance significativement plus grande que la moyenne) et le nombre d'unités d'échantillonnage petit (inférieur à 30 ), une transformation logarithmique a été appliquée aux données. L'ensemble des résultats est inscrit dans le tableau I.

Selon la méthode employée et le nombre d'individus récoltés, les limites de confiance sont plus ou moins acceptables :

- les résultats obtenus avec le filet de type Surber ne sont pas toujours interprétables, en particulier lorsque le nombre d'individus est inférieur à 10 .

Par contre, s'il est voisin de 100 , l'intervalle de confiance est relativement étroit.

- en utilisant un carottier avec 10 unités d'échantillonnage, les limites de confiance calculées sont assez resserrées; tandis qu'avec seulement 3 unités d'échantillonnage (aux stations $\mathbf{5 1}$ et $5 \mathrm{c}$ ) aucun résultat n'est interprétable.

Plusieurs auteurs ont déjà souligné l'influence de la dimension des relevés sur l'efficacité de l'échantillonnage (Elliott et Décamps 1973) lorsque la dispersion de la population est du type contagieux : un grand nombre de relevés de petite dimension étant la méthode la plus précise et la plus représentative.

Cependant, s'il est impossible de multiplier les unités d'échantillonnage, il vaut mieux employer un appareil dont la surface de base délimitera une aire assez importante (comparer les résultats obtenus aux stations 51 et 5 c avec 3 unités d'échantillonnage en employant un Surber de $300 \mathrm{~cm}^{2}$ et un carottier de $13 \mathrm{~cm}^{2}$ ) afin d'obtenir un intervalle de confiance relativement étroit.

\section{3. - LE MILIEU ETUDIÉ}

Le ruisseau étudié entaille le socle calcaire sur une vingtaine de kilomètres ; son cours monotone (pente de 7 à $3 \%$ ) est jalonné par des biefs de moulin. 


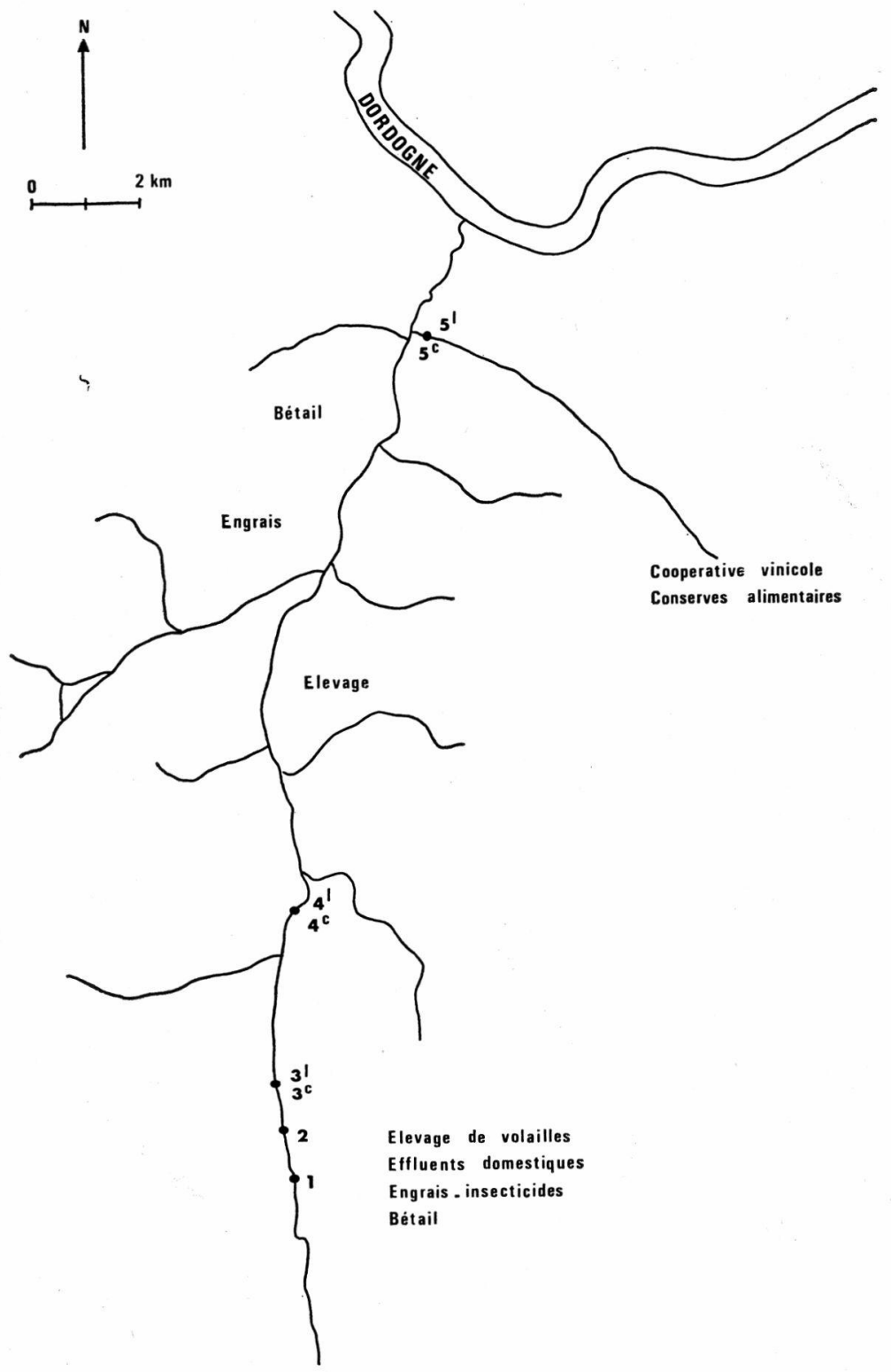

Fig. 1. - Principales agressions subies par le ruisseau. Emplacement des stations. 
niver

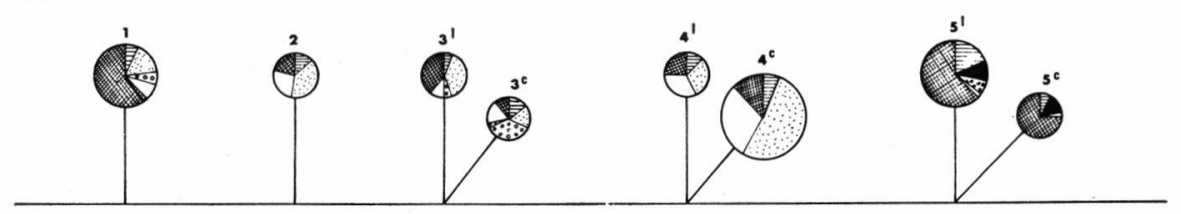

PRINTEMPS
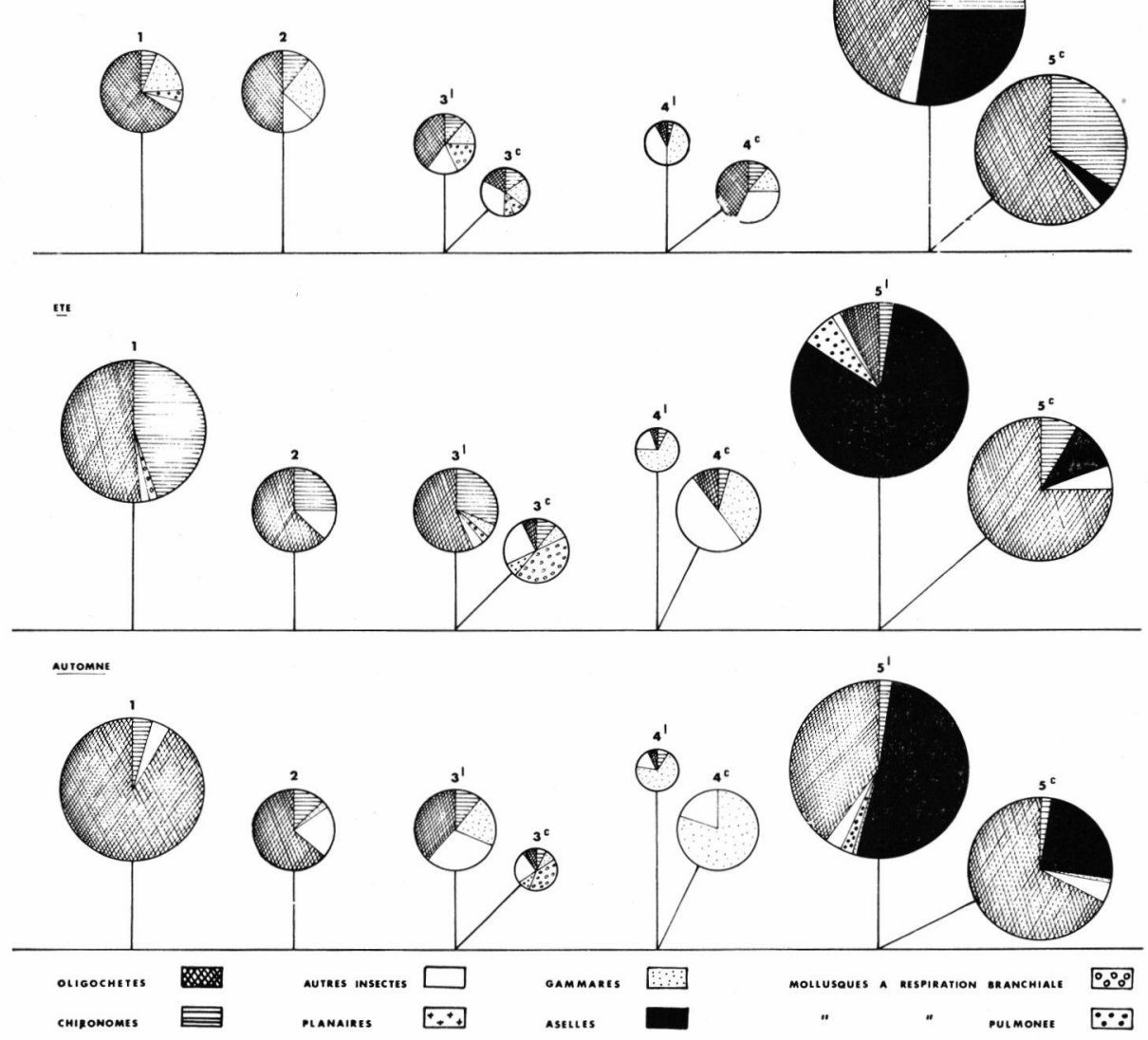

Fig. 2. - Abondance relative saisonnière des principaux groupes benthiques récoltés à chaque station.

L'activité humaine y provoque différentes agressions (voir fig. 1). Huit stations (fig. 1) ont été étudiées du point de vue physicochimique et faunistique : $\mathbf{2}$ en faciès lotique, blocs calcaires recouverts de bryophytes : station $3 \mathrm{c}$ et $4 \mathrm{c}$ (ou milieu a) ; 6 en courant faible à moyen sur substrat argilo-vaseux (ou milieu b). 
Les résultats physico-chimiques, outre des valeurs dues au socle calcaire $(\mathrm{pH}$ alcalin, conductivité électrique forte, sels de calcium très abondants, alcalinité forte) mettent en évidence :

- une température de l'eau qui reste basse et stable aux stations proches des sources (stations 2,3 et 4 ) ;

- un pourcentage de saturation en oxygène satisfaisant dans toutes les stations en hiver mais traduisant une situation douteuse ou critique en période d'étiage aux stations $1,2,3$ et 5 .

- des taux de chlorures, sulfates, orthophosphates, nitrates et nitrites traduisant une pollution insidieuse sur tout le cours du ruisseau, faible à la station 4 , augmentant aux stations 3 , 2 et 1 , forte à la station 5 .

\section{4. - LES COMMUNAUTÉS D'OLIGOCHẼTES}

\section{1. - Résultats.}

L'ensemble des résultats (fig. 2) montre une transformation progressive des communautés d'invertébrés benthiques des stations propres (stations 41 et $4 \mathrm{c}$ ) aux stations polluées organiquement (stations 51 et $5 \mathrm{c}$ ). Les communautés les plus diversifiées ne comptent qu'un petit nombre d'individus par $\mathbf{0 , 1} \mathrm{m}^{2}$ tandis que les stations perturbées renferment des communautés simplifiées où prolifèrent deux ou trois espèces connues pour leur résistance à la pollution organique.

Les graphiques de la figure 3 traduisent l'évolution saisonnière des communautés d'Oligochètes à chaque station. Il a été précisé entre parenthèses, pour chaque prélèvement, la densité représentée par la population d'Oligochètes dans les communautés d'invertébrés benthiques.

Les Oligochètes récoltés appartiennent aux trois familles : Tubificidae, Naïdidae et Lumbriculidae; les déterminations ont été faites jusqu'à l'espèce seulement pour la famille des Tubificidae. Sur le diagramme, les trois espèces de Limnodrilus : hoffmeisteri (Claparède), udekemanius (Claparède) et claparedeanus (Ratzel) (Cette dernière trouvée uniquement à la station 5) ont été représentées ensemble par $\Sigma$ Limnodrilus.

Une comparaison entre les populations d'Oligochètes des différentes stations n'est possible que si l'on tient compte du substrat :

- les communautés d'Oligochètes colonisant le milieu a (faciès lotique) sont formées essentiellement de Naïdidae et Lumbriculidae, le seul Tubificidae se développant dans ces stations étant Psammoryctides barbatus (Grube). 
Il y a en général peu d'individus (en moyenne 30 ind./0,1 $\mathrm{m}^{2}$ ), sauf au printemps en $4 \mathrm{c}$, le prélèvement correspondant à l'éclosion des Naïdidae; la communauté d'Oligochètes est très peu importante par rapport à l'ensemble de la communauté d'invertébrés benthiques sauf au printemps en $4 c$ (même remarque que précédemment).

- sur substrat argilo-vaseux se développent essentiellement des Tubificidae. Le nombre d'individus est toujours important $\left(\simeq 2000\right.$ ind. $\left./ 0,1 \mathrm{~m}^{2}\right)$; les Oligochètes représentent un fort pourcentage de la population totale (supérieur à $30 \%$ ) sauf en 41 où l'essentiel de la communauté est représenté par des Gammares et en 51 été (fig. 2).

Les communautés d'Oligochètes sont diversifiées dans les quatre premières stations et simplifiées aux stations $5 \mathrm{l}$ et $5 \mathrm{c}$; les espèces Tubifex tubifex (Müller), Limnodrilus udekemanius et L. hoffmeisteri colonisent toutes les stations et sont généralement représentées par un grand nombre d'individus tandis que les espèces Psammoryctides barbatus, Tubifex ignotus (Stolc), Aulodrilus pluriseta (Pignet) et Potamothrix hammoniensis (Michaelsen) ont des densités fluctuantes.

Des individus sexués de grande taille appartenant aux espèces L. hoffmeisteri, L. udekemanius et Tubifex tubifex ont été trouvés tout au long de l'année aux stations 1, 51 et 5c. A la station 31, $L$. hoffmeisteri et $L$. udekemanius ont une période de reproduction très courte, au début de l'automne, celle de Tubifex tubifex se situant au printemps.

\section{2. - Discussion.}

La disparité entre les populations d'Oligochètes trouvées dans les deux types de milieux a été mise en évidence par différents auteurs en particulier Brinkhurst et Kennedy (1962) : le biotope a (stations 3c et 4c) rappelle le rhithron (les ruisseaux à truites) où Brinkhurst (1966 a et b) note la présence de Lumbriculidae et Naïdidae, les Tubificidae y étant en général mal représentés si l'apport en matière organique n'est pas trop important.

Sur substrat argilo-vaseux, la communauté la plus fréquemment représentée se compose de Tubifex tubifex, $L$. hoffmeisteri et $L$. udekemanius, association caractéristique des eaux polluées à faible pourcentage de saturation en oxygène (Kennedy 1965 ; Brinkhurst 1962, 1969 ; Timm 1970 ; Aston 1971, Ladle 1971, Milbrink 1973).

Cette communauté est pratiquement la seule à se développer aux stations 51 et $5 \mathrm{c}$ dont le substrat est formé d'une vase organique très épaisse et dont les caractéristiques physico-chimiques ont déjà mis en évidence (Gross 1975) une pollution due aux effluents domestiques et industriels (cave coopérative - usine de conserves 

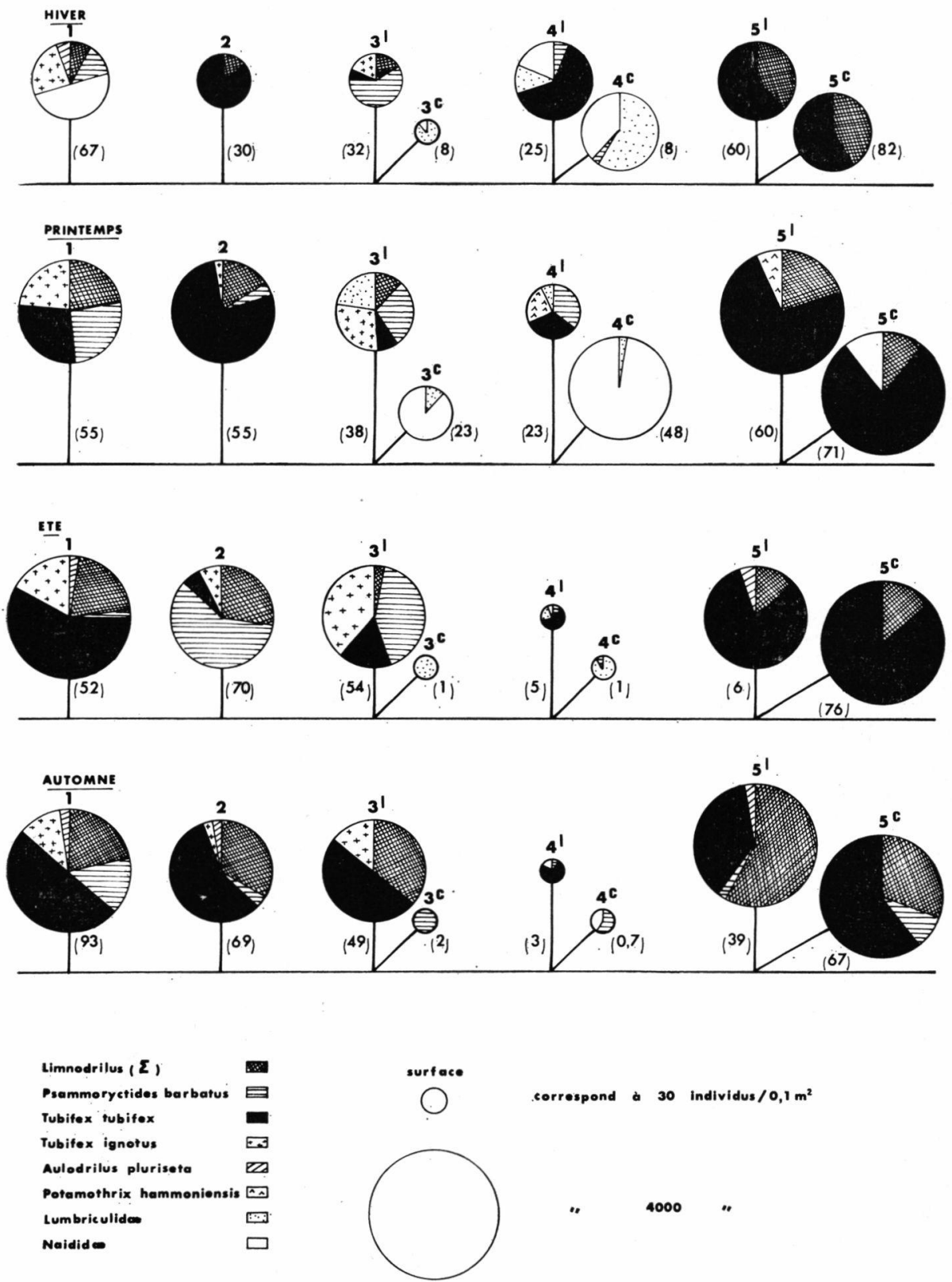

Fig. 3. - Evolution spatio-temporelle des communautés d'Oligochètes. Le pourcentage d'Oligochètes dans la communauté benthique a été précisé pour chaque prélèvement, entre parenthèses. 
alimentaires). Kennedy (1966 a et b) a montré que L. hoffmeisteri et $\boldsymbol{L}$. udekemanius pourraient avoir un cycle de reproduction s'étalant sur toute l'année dans les milieux très productifs (à teneur en matière organique forte) et à température élevée ; il n'est donc pas étonnant d'avoir trouvé aux stations 51 et $5 \mathrm{c}$ des individus sexués dans chaque prélèvement.

A l'association à Tubifex tubifex, L. hoffmeisteri et L. udekemanius s'ajoute très souvent Potamothrix hamnoniensis considérée comme tolérante vis-à-vis de la pollution organique (Brinkhurst et Jamieson 1971). La réunion de ces quatre espèces a déjà été notée en milieux pollués aussi bien lacs (Milbrink 1973) que rivières (Timm 1970).

Dans les stations 1,2 et 31 , outre les espèces déjà citées, on trouve fréquemment Tubifex ignotus et Psammoryctides barbatus ; à $T$. ignotus est souvent liée la notion de pollution (Milbrink $1973 \mathrm{~b}$ ) ; par contre $P$. barbatus est considérée par beaucoup d'auteurs comme sensible à un défaut d'oxygène et fréquentant davantage des substrats sableux que vaseux (Brinkhurst 1962, Juget 1958, Lastockin 1927).

Cependant, cette espèce est présente sur tout le cours du ruisseau quels que soient le substrat et la vitesse du courant. Sa présence semble être ici indépendante du taux de pollution organique et plutôt liée à la forte teneur en calcaire.

Il faut noter la présence d'Aulodrilus pluriseta à partir de l'été dans les stations 1 et 51 puis également à la station 2 en automne, stations où les problèmes posés par la pollution organique tout au long de l'année (Gross 1975) sont accentués par l'étiage.

On a peu de renseignements sur l'écologie de cette espèce si ce n'est que ses préférences écologiques rappellent celles de Potamothrix (Juget 1958) et qu'elle a été trouvée sur substrat de débris végétaux (Brinkhurst 1964 a).

\section{5. - UTILISATION DES OLIGOCHẼTES}

\section{COMME INDICATEURS DE LA QUALITÉ BIOLOGIQUE DE L'EAU}

Bien qu'il soit difficile de réaliser une étude quantitative très stricte - les sources d'erreurs sont nombreuses aussi bien dans l'application de la méthode d'échantillonnage que dans le tri une étude de ce type peut fournir des renseignements précis sur la qualité biologique d'un cours d'eau à condition de se baser sur un assez grand nombre de résultats.

Le calcul du pourcentage d'Oligochètes dans l'ensemble de la communauté benthique (voir tableau II) permet de classer les stations en deux groupes : 
- le $1^{\text {er }}$ groupe comprenant les stations où le pourcentage d'Oligochètes est inférieur à $20 \%$ : stations 3c, 4c et 41.

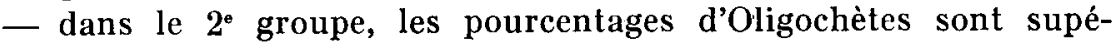
rieurs ou égaux à $40 \%$ avec un maximum autour de $70 \%$. Notons que la valeur moyenne $40 \%$ enregistrée en 51 est anormalement faible; elle est due à la valeur $6 \%$ enregistrée en été ; la densité moyenne de cette station se situerait plutôt aux alentours de $50 \%$ vus les résultats enregistrés aux autres saisons.

\begin{tabular}{|c|c|c|c|c|c|c|c|c|}
\hline \multirow{2}{*}{$\begin{array}{c}\text { Substrat } \\
\begin{array}{c}\text { Stations } \\
\text { I }\end{array}\end{array}$} & \multicolumn{2}{|c|}{$\begin{array}{c}\text { Pierres } \\
\text { bryophytes }\end{array}$} & \multicolumn{6}{|c|}{ Argilo-vaseux } \\
\hline & $\begin{array}{c}3^{\mathrm{c}} \\
10\end{array}$ & $\begin{array}{l}4^{\mathrm{e}} \\
14\end{array}$ & $\begin{array}{l}1 \\
68\end{array}$ & $\begin{array}{l}2 \\
56\end{array}$ & $\begin{array}{l}3^{1} \\
42\end{array}$ & $\begin{array}{l}4^{1} \\
14\end{array}$ & $\begin{array}{l}5^{1} \\
40\end{array}$ & $\begin{array}{l}5^{c} \\
74\end{array}$ \\
\hline $\begin{array}{l}\text { Psammoryctides barbatus } \\
\text { Limnodrilus } \\
\text { Tubifex tubifex } \\
\text { Tubifex ignotus } \\
\text { Aulodrilus pluriseta } \\
\text { Potamothrix hammoniensis } \\
\text { Lumbriculidae } \\
\text { Naididae }\end{array}$ & + & $\begin{array}{l}+ \\
+ \\
\end{array}$ & $\begin{array}{l}+ \\
+ \\
+ \\
+ \\
+\end{array}$ & $\begin{array}{l}+ \\
+ \\
+ \\
+ \\
+\end{array}$ & $\begin{array}{l}+ \\
+ \\
+ \\
+ \\
+ \\
+\end{array}$ & $\begin{array}{l}+ \\
+ \\
+ \\
+ \\
+\end{array}$ & $\begin{array}{l}+ \\
+ \\
+ \\
+ \\
+\end{array}$ & $\begin{array}{l}+ \\
+ \\
+\end{array}$ \\
\hline III & 0 & 0 & 70 & 71 & 41 & 0 & 96 & 95 \\
\hline
\end{tabular}

Tableau II. - I. Pourcentage moyen d'Oligochètes dans la communauté bentique. II. Répartition des espèces d'Oligochètes à chaque station (seule la présence est mentionnée ici, par une croix. III. Densité moyenne de la communauté indicatrice dans la population d'Oligochètes.

D'après Goodnight et Whitley (1960) une densité d'Oligochètes supérieure à $80 \%$ de la faune totale des macroinvertébrés traduit une forte pollution organique tandis qu'une densité comprise entre 60 et $80 \%$ caractérise des conditions douteuses.

Mais une étude quantitative de ce type ne peut suffire seule pour donner un diagnostic sur l'état du ruisseau. Elle doit être accompagnée d'une étude permettant de connaître la diversité spécifique de la communauté d'Oligochètes (voir résumé sur le tableau II).

Les différences de peuplements en Tubificidae d'une part et Naïdidae-Lumbriculidae d'autre part étant principalement liées au substrat (voir paragraphe 4-2), nous mettrons à part les stations 3c et $4 \mathrm{c}$.

Parmi les Tubificidae, nous avons vu que les espèces les plus tolérantes à la pollution organique étaient Tubifex tubifex, L. hoffmeisteri et L. udekemanius. Tubifex seule ne peut être considérée comme une espèce indicatrice (Brinkhurst 1964 b ; Dzwillo 1967).

Par contre, le calcul de la densité représentée par la communauté 
à Tubifex tubifex, L. hoffmeisteri et L. udekemanius (indicatrice de pollution organique) dans la population totale d'Oligochètes (voir tableau II) permet d'effectuer un classement des stations à substrat argilo-vaseux.

— La densité enregistrée en 41 est égale à 0 , la communauté indicatrice ne trouve pas un milieu favorable pour se développer : l'apport en matières organiques y est insuffisant; le pourcentage d'Oligochètes dans l'ensemble de la communauté benthique est faible.

Ces deux résultats permettent de dire que la station 41 n'est pas polluée organiquement.

- A l'inverse, aux stations 51 et 5 c se développe presque exclusivement la communauté indicatrice (96 et $95 \%$ ) ; les Oligochètes représentent un pourcentage important de la communauté d'invertébrés.

Ces deux stations subissent une pollution organique forte.

- Entre ces deux extrêmes se situent les stations 1 et 2 où la population d'Oligochètes est importante, la densité représentée par les espèces tolérantes est forte : à ces deux stations se développe une pollution organique assez forte variable selon les saisons.

La station 31 possède un pourcentage d'Oligochètes moyen, la communauté indicatrice a également une densité moyenne : la pollution organique commence à se développer dans cette station.

Un retour à la figure 2 montre plus précisément que les espèces tolérantes ont une forte densité surtout en automne : la station après l'étiage n'arrivant plus à retrouver le pouvoir autoépurateur qu'elle possédait en hiver - printemps.

Le même phénomène peut être enregistré à la station 1 : la détérioration de la situation $\mathrm{du}$ fait de la diminution du débit étant mise en évidence par l'évolution de la communauté indicatrice.

En résumé, sur le tableau III sont indiqués les pourcentages et densité par ordre croissant et un diagnostic sur l'état de pollution organique de chaque station.

Pourcentage d'Oligochètes dans

la communauté benthique

14

42

$56 \quad 68$

74

Densité de la communauté

indicatrice

$0 \quad 4$

Stations

41

41

71

70

95

Pollution organique

nulle faible

$2 \quad 1$

5

moyenne à assez forte

forte

TABLEaU III. - Synthèse des données : évolution de la pollution organique selon les stations sur substrat argilo-vaseux. 


\section{6. - CONCLUSION}

Bien que cette étude n'ait porté que sur un seul ruisseau pendant un an, bien que notre méconnaissance actuellement de la biologie de certaines espèces et de la répartition spatiale des individus au sein d'un habitat puissent provoquer des erreurs d'interprétation, l'étude des communaulés d'Oligochètes semble pouvoir être utilisable dans la détermination de la qualité biologique de l'eau.

Cette étude comprend trois étapes :

1. Calcul du pourcentage d'Oligochètes dans l'ensemble de la population d'invertébrés benthiques ;

2. Recherche, dans la communauté d'Oligochètes, de la diversité spécifique et du nombre d'individus de chaque espèce.

3. Suivie des variations de densité de la communauté à Tubifex tubifex, Limnodrilus hoffmeisteri et Limnodrilus udekemanius indicatrice de pollution organique au sein de la communauté d'Oligochètes; observation des cycles de reproduction de ces espèces in situ.

La confrontation entre ces trois groupes de résultats permet de déterminer l'existence et l'évolution de la pollution organique dans une série de stations sur substrat argilo-vaseux; les stations où ne se développe pas la communauté indicatrice et où le pourcentage d'Oligochètes dans la population benthiques est faible étant considérées comme non polluées organiquement.

Puis, le pourcentage d'Oligochètes (I) et la densité de la communauté indicatrice (III) augmentent avec la pollution.

Les stations ayant un pourcentage d'Oligochètes supérieur à $50 \%$ et une densité de la communauté indicatrice inférieure à $50 \%$ subissent une pollution organique moyenne.

Les stations à pourcentage d'Oligochètes supérieur à $70 \%$ et une densité (III) supérieure à $90 \%$ subissent une pollution organique forte.

\section{TRAVAOX GITES}

Aston (R. J.). 1971. - The effects of temperature and dissolved oxygen concentration on reproduction in Limnodrilus hoffmeisteri (Claparède) and Tubifex tubifex (Müller) Oligochaeta Tubificidae. C.E.R.L. Lab. Note RD/L/M 312 (Central Electric Research Laboratories).

Brinkhurst (R. O.) et Kennedy (C. R.). 1962. - Some aquatic Oligochaeta from the Isle of Man with special reference to the Silver Burn Estuary. Arch. Hydrobiol., 58 : 367-376. 
BrinkmuRst (R. O.). 1964 a. - Observations on the biology of the Tubificidae (Oligochaeta). Verh. Int. Verein ther. angew Limnol., $15,855-863$.

Brinkhurst (R, O.). $1964 \mathrm{~b}$. - Observations on the biology of lake dwelling Tubificidae (Oligochaeta), Arch. Hydrobiol., 60 : 385-418.

Brinkhurst (R. O.). 1966 a. - The Tubificidae (Oligochaetae) of polIuted water. Verh. Int. Verein Theor. angew Limnol., 16 : 854-959.

BrinkmRst (R. O.). 1966 b. - Detection and assessment of water pollution using Oligochaeta worms. WHO/EBL Report.

Brinkmurst (R. O.). 1969. - Changes in the benthos of lakes Erie and Ontario. Proc. Conf. Changes in the Biota of Lakes Erie and Ontario. Bull. Buffalo. Soc. Nat. Sei., $25: 45-65$.

Brinkhurst (R. O.) et Jamieson (B. G. M.). 1971. - Aquatic Oligochaeta of the World; Oliver and Boyd, $860 \mathrm{pp}$.

Dzwillo (M.). 1967. - Untersuchungen über die Zusammensetzung der Tubificidenfauna in Bereich des Hamburger Hafens. Verh. Naturw. Ver. Hamburg, N.F.. XI : 101-116.

Erliott (J. M.) et Décamps (H.). 1973. - Guide pour l'analyse statistique des échantillons d'invertébrés benthiques. Annls. Limnol., 9 (2) : 79-120.

Goodnight (C. J.) et Whit LEy (L. S.). 1960. - Oligochaetes as indicators of pollution. Wat. Sewage Wks., 107-311.

Gross (F.). 1975. - Etude écologique d'un ruisseau de l'Entre-Deux-Mers. Thèse de spécialité. $164 \mathrm{pp}$. Université de Bordeaux I.

Gross (F.). 1976. - Appréciation de la qualité biologique d'un cours d'eau de plaine. Bulletin français de pisciculture, $\mathrm{n}^{\circ} 260$ (à paraître).

Juget (J.). 1958, - Recherche sur la faune de fond du Léman et du lac d'Annecy. Annls Sin. Cent. Hydrobiol appl., $7: 9-96$.

KenNeDY (C. R.). 1965. - The distribution and habitat of Limnodrilus Claparède (Oligochaeta, Tubificidae). Oikos, $16: 26-38$.

KENNEDY (C. R.). 1966 a. - The life history of Limnodrilus udekemanius Clap, (Oligochaeta, Tubificidae). Oikos, 17, 10-18.

KenNEDY (C. R.). 1966 b. - The life history of Limnodrilus hoffmeisteri Clap (Oligochaeta, Tubificidae) and its adaptive significance. Oikos, 17, 158-168.

Kolkwitz (R.) et MARsson (M.). 1908. — ökologie der Pflanzischen Saprobien. Ber. deutsch. Bot. Ges., 26 :505-519.

Kolkwitz (R.) et Marsson (M.). 1909. - ökologie der tierischen Saprobien. Ent. Rev. Ges. Hydrograph., $2: 826-852$.

LadeE (M.). 1971. - The biology of Oligochaeta from Dorset chalk streams. Freshwat. Biol., $1: 83-97$.

Lastockin (D. A.). 1927. - Beiträge zu Oligochactan Russlands, III. Bull. Inst. Polyt. Iran Vosensensk, $10: 65-76$.

Milbrink (G.). 1973 a. - Communities of Oligochaeta as indicators of the water quality in lake Hjälmaren. Zoon, Swed., $1: 77-88$.

Milbrink (G.). 1973 b. - On the use of indicator communities of Tubificidae and some Lumbriculidae in the assessment of water pollution in Swedish lakes. Zoon, Swed, 1 (2) : 125-139.

Shannon (C. E.). 1948, - A mathematical theory of communication. Bell. Syst. Tech. J., $27: 379-423,623-656$.

Tाмm (T.). 1970. - On the fauna of the Estonian Oligochaeta. Pedobiologia, $10: 52-78$.

Tuffery (G.) et Verneaux (J.). 1967. - Méthodes de détermination de la qualité biologique des eaux courantes. Exploitation codifiée des inventaires de la faune du fond. Trav. Div. Qual. Eaux P. Pisc. C.T.G.R.E.F. Paris, 23 pp. 